\title{
Note on the point-splitting procedure to evaluate vacuum fluctuation in certain cylindrically symmetric backgrounds
}

\author{
I. Brevik* \\ Department of Applied Mechanics, Norwegian University of Science and Technology, N-7034 Trondheim, Norway \\ G. E. A. Matsas ${ }^{\dagger}$ and E. S. Moreira, Jr. ${ }^{\dagger}$ \\ Instituto de Física Teórica, Universidade Estadual Paulista, Rua Pamplona 145, 01405-900, São Paulo, São Paulo, Brazil
}

(Received 26 March 1998; published 23 June 1998)

\begin{abstract}
We reexamine the two-point function approaches used to study vacuum fluctuation in wedge-shaped regions and conical backgrounds. The appearance of divergent integrals is discussed and circumvented. The issue is considered in the context of a massless scalar field in cosmic string spacetime. [S0556-2821(98)03814-4]
\end{abstract}

PACS number(s): 04.70.Dy, 04.62.+v

\section{INTRODUCTION}

The vacuum fluctuation is one of the most exquisite features of quantum field theory and has been extensively studied since Casimir's seminal work [1]. Two-point functions are particularly useful to analyze vacuum properties [2,3]. The Hadamard function $G^{(1)}\left(x, x^{\prime}\right):=\left\langle\left\{\phi(x), \phi\left(x^{\prime}\right)\right\}\right\rangle$ gives comprehensive insight into such fluctuations. For example, $\left\langle\phi^{2}\right\rangle$ can be expressed as

$$
\left\langle 0\left|\phi^{2}(x)\right| 0\right\rangle=\lim _{x^{\prime} \rightarrow x} \frac{1}{2} G^{(1)}\left(x, x^{\prime}\right) .
$$

In some cases, however, it may be preferable to cast the formula above in terms of the Feynman propagator

$$
G_{\mathcal{F}}\left(x, x^{\prime}\right)=-\frac{i}{2} G^{(1)}\left(x, x^{\prime}\right)-\bar{G}\left(x, x^{\prime}\right),
$$

as

$$
\left\langle 0\left|\phi^{2}(x)\right| 0\right\rangle=i \lim _{x^{\prime} \rightarrow x} G_{\mathcal{F}}\left(x, x^{\prime}\right)+i \lim _{x^{\prime} \rightarrow x} \bar{G}\left(x, x^{\prime}\right),
$$

where $\bar{G}\left(x, x^{\prime}\right)$ is the average of the retarded and advanced Green functions. In particular $\bar{G}\left(x, x^{\prime}\right)$ vanishes if $x$ and $x^{\prime}$ are not causally connected [4], in which case Eq. (3) becomes

$$
\left\langle 0\left|\phi^{2}(x)\right| 0\right\rangle=i \lim _{x^{\prime} \rightarrow x} G_{\mathcal{F}}\left(x, x^{\prime}\right) .
$$

The present paper was triggered by the following mathematical problem. In the process of evaluating Eq. (4) in a variety of different situations, Deutsch and Candelas [5], Helliwell and Konkowski [6], and Matsas [7] have made use of the integration formula

\footnotetext{
*Email address: Iver.H.Brevik@mtf.ntnu.no

${ }^{\dagger}$ Email address: matsas@axp.ift.unesp.br

"Email address: moreira@axp.ift.unesp.br
}

$$
\int_{0}^{\infty} d p p^{1-\epsilon} J_{\nu}^{2}(p r)=\frac{2^{1-\epsilon} \Gamma(-1+\epsilon) \Gamma(\nu+1-\epsilon / 2)}{r^{2-\epsilon} \Gamma^{2}(\epsilon / 2) \Gamma(\nu+\epsilon / 2)}
$$

in the $\epsilon \rightarrow 0$ limit. However, as noted in Ref. [8] the integral above is convergent only when $\epsilon>1$; i.e., Eq. (5) is not valid in the region $\epsilon \rightarrow 0$. Quite analogously, an equivalent divergent integral [see Eq. (2.15.3-3) of Ref. [9]] was used in a similar context [see Eq. (17) of Ref. [10] for $N \geqslant 3$ ]. Rather surprisingly, however, a pretty different approach [8] based on Schwinger's source theory led to the same final results of Refs. [5-7,10]. As expressed in Sec. VI C of Ref. [8], "We have not got any explanation for the fact that although Deutsch and Candelas use (5) their final answer is correct.",

In this paper we reexamine the methods used in $[5-7,10]$, giving a more detailed explanation for this delicate behavior. For the sake of clarity we focus on the discussion of the vacuum fluctuation outside cosmic strings but our conclusions must be equally valid for other cylindrically symmetric backgrounds where the same problem appears. In Sec. II we discuss the issue using dimensional analytic continuation. In Sec. III we spot where regularization takes place. In Sec. IV a few remarks are considered and our conclusions are summarized.

\section{DIMENSIONAL ANALYTIC CONTINUATION}

During the past years there has been considerable interest in the study of the vacuum fluctuation outside cosmic strings (see $[6-8,10,11]$ and references therein). The line element of the spacetime outside a cosmic string in $N$ dimensions can be written in the form

$$
d s^{2}=d t^{2}-d r^{2}-r^{2} d \theta^{2}-d \mathbf{z}^{2}
$$

where $0 \leqslant \theta<\alpha:=2 \pi|1-4 \mu|$ and $\mathbf{z} \equiv\left(z_{1}, \ldots, z_{N-3}\right)$. For $N=4, \mu / G$ is the mass per unit length of the string. We are assuming an $N$-dimensional spacetime as this is the parameter which will be used to analytic-continue the Green function into four dimensions. 
Considering the identification $\theta \sim \theta+\alpha$ the Feynman propagator of a massless scalar field in this background can be written for $r=r^{\prime}$ and $\mathbf{z}=\mathbf{z}^{\prime}$ as [10]

$$
\begin{aligned}
D_{\mathcal{F}}^{(N, \alpha)}(\tau ; \Delta)= & \frac{-i}{\alpha} \int_{-\infty}^{+\infty} \frac{d \omega}{2 \pi} \int \frac{d^{N-3} \mathbf{k}}{(2 \pi)^{N-3}} \int_{0}^{\infty} d p p \int_{0}^{\infty} d s \\
& \times e^{-i s\left(p^{2}+\mathbf{k}^{2}-\omega^{2}\right)} e^{-i \omega \tau} \\
& \times \sum_{n=-\infty}^{\infty} J_{2 \pi|n| / \alpha}^{2}(p r) e^{i 2 \pi n \Delta / \alpha},
\end{aligned}
$$

where $\Delta:=\theta-\theta^{\prime}$ and $\tau:=t-t^{\prime}$. By setting $\tau=0$ (note that for $N=4$ one recovers the Feynman propagator in [6]) and making transformations $\omega \rightarrow-i \omega$ and $s \rightarrow i s$ followed by a Wick rotation back to the real axes, we perform the integrations over $\omega, \mathbf{k}$, and $s$, obtaining, for $N<4$ (see, e.g., Ref. [9]),

$$
\begin{aligned}
D_{\mathcal{F}}^{(N, \alpha)}(\Delta)= & \frac{-i \pi^{1-N / 2} \Gamma(2-N / 2)}{2^{N-2} \alpha} \\
& \times \sum_{n=-\infty}^{\infty} e^{i 2 \pi n \Delta / \alpha} \int_{0}^{\infty} d p p^{N-3} J_{2 \pi|n| / \alpha}^{2}(p r) .
\end{aligned}
$$

For $N=4$ the integral that appears above is the divergent one in Eq. (5). Notwithstanding, it is convergent in the interval $2<N<3$, leading to

$$
\begin{aligned}
D_{\mathcal{F}}^{(N, \alpha)}(\Delta)= & \frac{-i \Gamma[(3-N) / 2]}{\alpha(2 \sqrt{\pi})^{N-1} r^{N-2}} \\
& \times \sum_{n=-\infty}^{\infty} \frac{\Gamma(-1+N / 2+2 \pi|n| / \alpha)}{\Gamma(2-N / 2+2 \pi|n| / \alpha)} e^{i 2 \pi n \Delta / \alpha},
\end{aligned}
$$

in agreement with Ref. [10]. Assuming now that both sides of Eq. (9) are analytic functions of $N$, by the principle of analytic continuation equality (9) also holds for $N \geqslant 3$. Thus after performing the summation with $N=4$ one obtains

$$
D_{\mathcal{F}}^{(4, \alpha)}(\Delta)=-\frac{i}{4 \alpha^{2} r^{2}} \csc ^{2} \frac{\pi \Delta}{\alpha}
$$

which is the correct result as has been rigorously shown in Ref. [8].

The reason why we (and the authors of Refs. [5-7,10]) have arrived at the correct result may be traced back to Eq. (8). Namely, the divergent integral on the right-hand side (RHS) of Eq. (8) is regularized by the summation. Thus, although the integral itself is not analytic when $N=4$ since it is divergent, the RHS of Eq. (8) as a whole is analytic. In the next section we will show how the divergence can be isolated and explicitly regularized.

\section{REGULARIZATION PROCEDURE}

The regularization procedure adopted in this section will be possible by considering, in Eq. (7), $\tau \neq 0$ (eventually the limit $\tau \rightarrow 0$ will be taken) and that the interval associated with $\left(x, x^{\prime}\right)$ is spacelike [then vacuum expectation values can be extracted from the Feynman propagator as in Eq. (4)]. This can be implemented by using an approach slightly different from the one above.

Performing integrations in Eq. (7) except over the $s$ variable we obtain [10]

$$
\begin{aligned}
D_{\mathcal{F}}^{(N, \alpha)}(\tau ; \Delta)= & \frac{2 \pi}{\alpha} \int_{0}^{\infty} \frac{d s}{(4 \pi i s)^{N / 2}} e^{-i\left(\tau^{2}-2 r^{2}\right) / 4 s} \\
& \times \sum_{n=-\infty}^{\infty} I_{2 \pi|n| / \alpha}\left(r^{2} / 2 i s\right) e^{i 2 \pi n \Delta / \alpha}
\end{aligned}
$$

The remaining integral above can be evaluated by first adding an infinitesimal negative imaginary part to $\tau^{2}$ and then by using [9]

$$
\begin{aligned}
\int_{0}^{\infty} d x x^{\mu-1} e^{-p x} I_{\nu}(c x) \\
=p^{-(\mu+\nu)\left(\frac{c}{2}\right)^{\nu} \frac{\Gamma[\nu+\mu]}{\Gamma[\nu+1]}} \\
\quad \times_{2} F_{1}\left[\frac{\mu+\nu}{2}, \frac{\mu+\nu+1}{2} ; \nu+1 ; \frac{c^{2}}{p^{2}}\right],
\end{aligned}
$$

which holds for $\operatorname{Re}(\mu+\nu)>0$ and $\operatorname{Re} p>|\operatorname{Re} c|$. The hypergeometric function in Eq. (12) can be recast by considering a linear transformation (see, e.g., Ref. [12])

$$
\begin{aligned}
{ }_{2} F_{1}[a, b ; c ; z] \\
=\frac{\Gamma[c] \Gamma[c-a-b]}{\Gamma[c-a] \Gamma[c-b]} \\
\quad \times{ }_{2} F_{1}[a, b ; 1+a+b-c ; 1-z]+\frac{\Gamma[c] \Gamma[a+b-c]}{\Gamma[a] \Gamma[b]} \\
\quad \times{ }_{2} F_{1}[c-a, c-b ; 1-a-b+c ; 1-z](1-z)^{c-a-b},
\end{aligned}
$$

whose domain of validity is the $z$ plane cut along the real axis from $z=-\infty$ to $z=0$ and from $z=1$ to $z=\infty$, with

$$
a+b-c \neq 0, \pm 1, \pm 2, \ldots .
$$

Thus the Feynman propagator can be written as shown in Eq. (2) with 


$$
\begin{aligned}
D^{(1)(N, \alpha)}(\tau ; \Delta)= & \frac{1}{\alpha} \sum_{n=-\infty}^{\infty} e^{i 2 \pi n \Delta / \alpha} \frac{\pi^{(1-N) / 2} r^{2-N}}{2^{N-2}}\left(1-\frac{\tau^{2}}{2 r^{2}}\right)^{1-N / 2-2 \pi|n| / \alpha} \frac{\Gamma[-1+N / 2+2 \pi|n| / \alpha] \Gamma[(3-N) / 2]}{\Gamma[2-N / 2+2 \pi|n| / \alpha]} \\
& { }_{2} F_{1}\left[\frac{\pi|n|}{\alpha}+\frac{N-2}{4}, \frac{\pi|n|}{\alpha}+\frac{N}{4} ; \frac{N-1}{2} ; 1-\left(1-\frac{\tau^{2}}{2 r^{2}}\right)^{-2}\right]
\end{aligned}
$$

and

$$
\begin{aligned}
\bar{D}^{(N, \alpha)}(\tau ; \Delta)= & \frac{i}{4 \alpha} \sum_{n=-\infty}^{\infty} e^{i 2 \pi n \Delta / \alpha} \pi^{(1-N) / 2} r^{2-N}\left(1-\frac{\tau^{2}}{2 r^{2}}\right)^{1-N / 2-2 \pi|n| / \alpha} \\
& \times\left[1-\left(1-\frac{\tau^{2}}{2 r^{2}}\right)^{-2}\right]^{(3-N) / 2} \Gamma\left(\frac{N-3}{2}\right){ }_{2} F_{1}\left[\frac{|n| \pi}{\alpha}+\frac{6-N}{4}, \frac{|n| \pi}{\alpha}+\frac{4-N}{4} ; \frac{5-N}{2} ; 1-\left(1-\frac{\tau^{2}}{2 r^{2}}\right)^{-2}\right] .
\end{aligned}
$$

Using these expressions for small $\tau$, the Feynman propagator becomes

$$
D_{\mathcal{F}}^{(N, \alpha)}(\tau ; \Delta)=\frac{-i \Gamma[(3-N) / 2]}{\alpha(2 \sqrt{\pi})^{N-1} r^{N-2}} \sum_{n=-\infty}^{\infty} \frac{\Gamma(-1+N / 2+2 \pi|n| / \alpha)}{\Gamma(2-N / 2+2 \pi|n| / \alpha)} e^{i 2 \pi n \Delta / \alpha}-\frac{i^{-N} \Gamma[(N-3) / 2]}{4 \pi^{(N-1) / 2} r|\tau|^{N-3}} \sum_{n=-\infty}^{\infty} \delta(\Delta+\alpha n),
$$

which holds for nonodd $N$ [as follows from Eq. (13)], $N$ $>2$. In obtaining Eq. (14) we have also used Poisson's formula

$$
\sum_{m=-\infty}^{\infty} \delta(\phi+2 \pi m)=\frac{1}{2 \pi} \sum_{m=-\infty}^{\infty} \exp \{\operatorname{im} \phi\}
$$

By taking $\tau \rightarrow 0$ in Eq. (14) when $N<3$ we recover Eq. (9) as it should be. Now when $N>3$ it may appear that we are left with a divergence as $\tau \rightarrow 0$ due to the factor $1 /|\tau|^{N-3}$ in the second term on the RHS of Eq. (14). However, before taking $\tau \rightarrow 0$, we should note that $\delta(\Delta+\alpha n)$ vanishes identically for every $n$ since $\Delta \neq \alpha n$ (recall that we are dealing with a spacelike interval). Thus the second term on the RHS of Eq. (14) is null and Eq. (9) still holds for $N>3$.

\section{FINAL REMARKS}

Before closing the paper let us discuss a couple of points. As mentioned above, Eq. (9) is not suitable for odd $N$. For example, when $N=3, \Gamma(0) \delta(\Delta)$ arises in Eq. (9) which needs to be regularized. Using $\Gamma[a+z] / \Gamma[b+z]=B[z$ $+a, b-a] / \Gamma[b-a]$ with $B[z, w]$ denoting the Euler beta function, we see that Eq. (9) may be rewritten as

$$
\begin{aligned}
D_{\mathcal{F}}^{(N, \alpha)}(\Delta)= & \frac{1}{i \alpha 2 \pi^{N / 2-1} r^{N-2} \Gamma[(4-N) / 2]} \\
& \times\left\{B\left[\frac{N-2}{2}, 3-N\right]+2 \sum_{m=1}^{\infty} B\left[\frac{2 \pi m}{\alpha}\right.\right. \\
& \left.\left.+\frac{N-2}{2}, 3-N\right] \cos \frac{2 \pi m \Delta}{\alpha}\right\},
\end{aligned}
$$

where we have used the fact that $\Gamma[2 x] / \Gamma[x]$ $=2^{2 x-1} \pi^{-1 / 2} \Gamma[x+1 / 2]$. By using $B[z, w]:=\int_{0}^{1} d t t^{z-1}(1$ $-t)^{w-1}$ in the second term in Eq. (15) and performing the summation [12], we are left with the following integral representation [13]:

$$
\begin{aligned}
D_{\mathcal{F}}^{(N, \alpha)}(\Delta)= & \frac{1}{i \alpha 2 \pi^{N / 2-1} r^{N-2} \Gamma[(4-N) / 2]} \\
& \times \int_{0}^{1} d t t^{(t-4) / 2} \frac{(1-t)^{2-N}\left(1-t^{4 \pi / \alpha}\right)}{1-2 t^{2 \pi / \alpha} \cos \{2 \pi \Delta / \alpha\}+t^{4 \pi / \alpha}},
\end{aligned}
$$

which is suitable for odd $N$ (but not for even $N$ ).

Finally let us consider a check of consistency. For $N=4$, which is our main interest here, one obtains, from Eq. (14),

$$
D_{\mathcal{F}}^{(4, \alpha)}(\tau ; \Delta)=-\frac{i}{4 \alpha^{2} r^{2}} \csc ^{2} \frac{\pi \Delta}{\alpha}-\frac{1}{4 \pi r|\tau|} \sum_{n=-\infty}^{\infty} \delta(\Delta+\alpha n) .
$$

The first term in Eq. (16) is the familiar expression (10). The second term, which vanishes, was kept just for a formal comparison with the usual Minkowski spacetime result. Indeed Eq. (16) with $\alpha=2 \pi$ must be consistent with the usual expression in Minkowski spacetime [2]:

$$
D_{\mathcal{F}}^{(4,2 \pi)}\left(x, x^{\prime}\right)=\frac{i}{8 \pi^{2} \sigma}-\frac{\delta(\sigma)}{8 \pi},
$$

where $2 \sigma:=\left|x^{\mu}-x^{\prime \mu}\right|^{2}$. Starting from Eq. (17) this fact can be verified by noting that (for $r=r^{\prime}$ and $\mathbf{z}=\mathbf{z}^{\prime}$ ) in cylindrical coordinates $2 \sigma=\tau^{2}-2 r^{2}+2 r^{2} \cos \Delta$. Then using the formula $\delta[f(x)]=\Sigma_{j} \delta\left(x-x_{j}\right) /\left|f^{\prime}\left(x_{j}\right)\right|$, where $x_{j}$ are the simple zeros of $f(x)$, it is rather straightforward to reproduce Eq. (16) in the limit of small $\tau$. 
In summary we have investigated the reason why a number of previous works have obtained the correct result for a vacuum fluctuation in wedge-shaped regions and conical backgrounds although using illicit expressions for divergent integrals. The problem is solved by noting that there is an overall summation which renders the result finite. In Sec. II we use the principle of analytic continuation to obtain a definite result, while in Sec. III such a principle is verified by isolating the divergence and regularizing. With these clarifi- cations in mind those works that have obtained correct answers using illegal intermediate steps will not have their results seen as incidental.

\section{ACKNOWLEDGMENTS}

G.M. and E.M. would like to acknowledge partial and full financial support, respectively, from Conselho Nacional de Desenvolvimento Científico e Tecnológico and Fundação de Amparo à Pesquisa do Estado de São Paulo.
[1] H. B. G. Casimir, Proc. K. Ned. Akad. Wet. 51, 793 (1948).

[2] N. D. Birrell and P. C. W. Davies, Quantum Fields in Curved Space (Cambridge University Press, Cambridge, England, 1982).

[3] S. A. Fulling, Aspects of Quantum Field Theory in Curved Space-time (Cambridge University Press, Cambridge, England, 1989).

[4] D. G. Boulware, Phys. Rev. D 11, 1404 (1975).

[5] D. Deutsch and P. Candelas, Phys. Rev. D 20, 3063 (1979).

[6] T. M. Helliwell and D. A. Konkowski, Phys. Rev. D 34, 1918 (1986).

[7] G. E. A. Matsas, Phys. Rev. D 41, 3846 (1990).

[8] I. Brevik and M. Lygren, Ann. Phys. (N.Y.) 251, 157 (1996).

[9] A. P. Prudnikov, Yu. A. Brychkov, and O. I. Marichev, Inte- grals and Series (Gordon and Breach, Amsterdam, 1986), Vol. 2.

[10] E. S. Moreira, Jr., Nucl. Phys. B451, 365 (1995).

[11] V. P. Frolov and E. M. Serebriany, Phys. Rev. D 35, 3779 (1987); B. Linet, ibid. 35, 536 (1987); Class. Quantum Grav. 9, 2429 (1992); J. S. Dowker, Phys. Rev. D 36, 3095 (1987); 36, 3742 (1987); A. G. Smith, in The Formation and Evolution of Cosmic Strings, Proceedings of the Cambridge Workshop, Cambridge, England, 1989, edited by G. W. Gibbons, S. W. Hawking, and T. Vachaspati (Cambridge University Press, Cambridge, England, 1990); G. E. A. Matsas, Phys. Rev. D 44, 2593 (1991); V. B. Bezerra, J. Math. Phys. 38, 2553 (1997).

[12] I. S. Gradshteyn and I. W. Ryzhik, Table of Integrals, Series, and Products (Academic, New York, 1980).

[13] E. S. Moreira, Jr., Ph.D. thesis, University of London, 1997. 